\title{
THE MODAL LOGIC OF AFFINE PLANES IS NOT FINITELY AXIOMATISABLE
}

\author{
IAN HODKINSON AND ALTAF HUSSAIN
}

\begin{abstract}
We consider a modal language for affine planes, with two sorts of formulas (for points and lines) and three modal boxes. To evaluate formulas, we regard an affine plane as a Kripke frame with two sorts (points and lines) and three modal accessibility relations, namely the point-line and line-point incidence relations and the parallelism relation between lines. We show that the modal logic of affine planes in this language is not finitely axiomatisable.
\end{abstract}

\$1. Introduction. Recently the modal logics of space have began to draw considerable interest from logicians and computer scientists. See, e.g., [1]. Much of the interest seems to stem from the perceived use of modal logics for qualitative reasoning about spatial relations between objects, and the potential applications in computer science and knowledge representation.

In this paper, we are concerned with the modal logics of projective and affine planes. In [2], geometries of points and lines were viewed as Kripke frames, the domain of each frame being the point-line incidence relation itself (i.e., the set of pairs $(s, l)$ where $s$ is a point on a line $l)$. A completeness theorem for 'incidence geometries' was proved, using a non-orthodox 'irreflexivity' inference rule, and extensions to projective and affine geometries were considered.

In [13], Venema viewed projective planes in a somewhat more straightforward way, as Kripke frames with two sorts (points and lines), and two modal accessibility relations (incidence between points and lines and between lines and points). $\mathrm{He}$ formulated a corresponding modal language with two sorts of formulas - point formulas (evaluated at points) and line formulas (at lines). He then presented a finite set of axioms, essentially expressing that the two accessibility relations are the converses of each other; every point lies on at least one line; any two points lie on at least one common line; and the duals of these two properties obtained by exchanging points and lines. The inference rules were orthodox: modus ponens, (well-sorted) substitution, and universal generalisation for each of the two sorts. Venema proved that the system is (strongly) sound and complete for projective planes. He also proved that the problem of determining whether a given formula is

Received June 14, 2007.

2000 Mathematics Subject Classification. Primary 51E15 Secondary 03B45, 51A15.

We thank the referee for helpful and interesting comments. The second author was supported by grant GR/S19004/01 from the U.K. EPSRC. 
satisfiable in some projective plane is decidable and complete for non-deterministic exponential time.

In [3], among many other things, Balbiani and Goranko regarded affine planes as two-sorted Kripke frames in a similar way, but with an additional accessibility relation relating parallel lines. They introduced a corresponding two-sorted modal language similar to Venema's, and proposed a finite set of axioms for affine planes in this language. Completeness of the axioms with respect to affine planes was left open (although it was proved for a wider class of structures called 'weak affine models').

In this paper, we will prove that in contrast to the case of projective planes, the modal logic of affine planes in this language is not finitely axiomatisable. This result first appeared in [9]. We should mention that in [10], Monk used affine planes in a rather similar way to prove that the variety of representable relation algebras is not finitely axiomatisable. We have borrowed some ideas (such as the use of the Bruck-Ryser theorem and compactness/ultraproducts) from Monk's proof. However, while the outline of our proof is similar to Monk's, the details are different. For example, propositions 3.7 and 3.8 below differ from their analogues in Monk's proof, and definition 3.1 and proposition 3.6 seem to have no analogue at all. Possibly a proof closer to Monk's can be found, or even a derivation of our result as a corollary of Monk's, or vice versa, but we have been unable to do this.

§2. Definitions. In this section, we recall the syntax and semantics of the twosorted modal language of [3] for affine planes, along with some other standard definitions and facts. We will prove the non-finite axiomatisability in section 3 .

\subsection{Syntax and semantics.}

Definition 2.1. We fix two disjoint, countably infinite sets $V A R_{p}$ of point variables and $V A R_{l}$ of line variables. The sets $\Pi$ and $\Lambda$ of point formulas and line formulas (respectively) of the modal language for affine planes are defined to be the smallest sets satisfying the following:

1. $V A R_{p} \subseteq \Pi$ and $V A R_{l} \subseteq \Lambda$.

2. If $\pi, \pi^{\prime} \in \Pi$ then $\neg \pi \in \Pi$ and $\pi \wedge \pi^{\prime} \in \Pi$.

3. If $\lambda, \lambda^{\prime} \in \Lambda$ then $\neg \lambda \in \Lambda$ and $\lambda \wedge \lambda^{\prime} \in \Lambda$.

4. If $\lambda \in \Lambda$ then [01] $\lambda \in \Pi$.

5. If $\pi \in \Pi$ then [10] $\pi \in \Lambda$

6. If $\lambda \in \Lambda$ then [11] $\lambda \in \Lambda$.

The numbers 0,1 are intended to suggest the relevant dimension $(0$ for points, 1 for lines). We adopt the usual abbreviations: if $\pi, \pi^{\prime}$ are point formulas, $\pi \vee \pi^{\prime}$ abbreviates $\neg\left(\neg \pi \wedge \neg \pi^{\prime}\right), \pi \rightarrow \pi^{\prime}$ abbreviates $\neg\left(\pi \wedge \neg \pi^{\prime}\right)$, and $\langle 10\rangle \pi$ abbreviates $\neg[10] \neg \pi$. Abbreviations for line formulas, and the diamonds $\langle 01\rangle$ and $\langle 11\rangle$, are defined similarly. The language is given semantics as follows.

Definition 2.2. A two-sorted affine frame (or simply a frame) is a two-sorted structure $\mathscr{F}=(P, L, \varepsilon, \|)$ such that $P \cap L=\emptyset, \varepsilon \subseteq P \times L$, and $\| \subseteq L \times L$. Elements of $P$ and $L$ are called points and lines, respectively; $\varepsilon$ is called the incidence relation; and $\|$ is called the parallel relation.

A valuation on $\mathscr{F}$ is a map $V: V A R_{p} \cup V A R_{l} \rightarrow \wp(P \cup L)$. 
A model is a pair $\mathscr{M}=(\mathscr{F}, V)$, where $\mathscr{F}$ is a frame and $V$ a valuation on $\mathscr{F}$. We sometimes write models in the form $(P, L, \varepsilon, \|, V)$, where $\mathscr{F}=(P, L, \varepsilon, \|)$. We define truth of formulas in such a model as follows. Let $s \in P$ and $l \in L$.

1. $\mathscr{M}, s \models v$ if $s \in V(v)$, for each $v \in V A R_{p}$.

2. $\mathscr{M}, l \models v$ if $l \in V(v)$, for each $v \in V A R_{l}$.

3. The boolean connectives are handled in the usual way.

4. $\mathscr{M}, s \models[01] \lambda$ if $\mathscr{M}, m \models \lambda$ for every $m \in L$ with $s \varepsilon m$.

5. $\mathscr{M}, l \models[10] \pi$ if $\mathscr{M}, t \models \pi$ for every $t \in P$ with $t \varepsilon l$.

6. $\mathscr{M}, l \models[11] \lambda$ if $\mathscr{M}, m \models \lambda$ for every $m \in L$ with $l \| m$.

Definition 2.3. A point formula $\pi$ is said to be valid in a frame $\mathscr{F}$ if we have $(\mathscr{F}, V), s \models \pi$ for every valuation $V$ on $\mathscr{F}$ and every point $s$ of $\mathscr{F} . \pi$ is said to be satisfiable in $\mathscr{F}$ if $\neg \pi$ is not valid in $\mathscr{F}$. Similar definitions are made for line formulas.

2.2. Bounded morphisms. We will need the notion of bounded morphism (cf. [4, definition 3.13]).

Definition 2.4. Let $\mathscr{F}=(P, L, \varepsilon, \|)$ and $\mathscr{F}^{\prime}=\left(P^{\prime}, L^{\prime}, \varepsilon^{\prime}, \|^{\prime}\right)$ be two-sorted affine frames. We say that $f: \mathscr{F} \rightarrow \mathscr{F}^{\prime}$ is a (surjective) homomorphism if $f: P \cup L \rightarrow$ $P^{\prime} \cup L^{\prime}$ is a (surjective) map with $f(s) \in P^{\prime}$ and $f(l) \in L^{\prime}$ for each $s \in P$ and $l \in L$, and the following Forth properties hold:

F1 If $s \varepsilon l$ then $f(s) \varepsilon^{\prime} f(l)$.

F2 If $l \| m$ then $f(l) \|^{\prime} f(m)$.

A homomorphism $f$ is said to be a bounded morphism if it additionally satisfies the following Back properties:

B1 If $s \in P, l^{\prime} \in L^{\prime}$, and $f(s) \varepsilon^{\prime} l^{\prime}$, then there is $l \in L$ with $s \varepsilon l$ and $f(l)=l^{\prime}$.

B2 If $l \in L, s^{\prime} \in P^{\prime}$, and $s^{\prime} \varepsilon^{\prime} f(l)$, then there is $s \in P$ with $s \varepsilon l$ and $f(s)=s^{\prime}$.

B3 If $l \in L, m^{\prime} \in L^{\prime}$, and $f(l) \|^{\prime} m^{\prime}$, then there is $m \in L$ with $l \| m$ and $f(m)=m^{\prime}$.

We say that $\mathscr{F}^{\prime}$ is a homomorphic image (respectively, a bounded morphic image) of $\mathscr{F}$ if there exists a surjective homomorphism (respectively, a surjective bounded morphism) $f: \mathscr{F} \rightarrow \mathscr{F}^{\prime}$.

REMARK 2.5. Any modal formula valid in a frame $\mathscr{F}$ is also valid in any bounded morphic image of $\mathscr{F}$. This is a standard fact for ordinary single-sorted modal logic, and the proofs in [4, theorem 3.14] and [6, corollary 2.16] easily generalise to our two-sorted frames.

2.3. Affine planes. An affine plane is a system of points and lines with an incidence relation between them, satisfying certain properties. For information, see, e.g., [8]. For convenience, we will state the classical definition in terms of our two-sorted affine frames.

Definition 2.6. A two-sorted affine frame $\mathscr{A}=(P, L, \varepsilon, \|)$ is said to be an affine plane if:

A1 For any two distinct points $s, t \in P$, there is exactly one line $l \in L$ such that $s \& l$ and $t \varepsilon l$. 
A2 For all $l, m \in L$, we have $l \| m$ iff $l=m$ or there is no $s \in P$ with $s \varepsilon l$ and $s \& m$.

A3 For any $l \in L$ and $s \in P$, there is exactly one line $m \in L$ such that $s \& m$ and $m \| l$.

A4 There are distinct $s, t, u \in P$ such that for no $l \in L$ do we have $s \varepsilon l, t \varepsilon l$, and $u \varepsilon l$.

The logic of affine planes is the set of all (point and line) formulas that are valid in every affine plane.

We will often use geometrical and set-theoretic language as shorthand to talk about frames. E.g., we will say that $s \varepsilon l \cap m$ if $s \varepsilon l$ and $s \varepsilon m$. In this language, the axioms for affine planes state that any two distinct points lie on a unique line, two lines are parallel iff they are equal or disjoint, there is a unique line through any given point parallel to any given line, and there exist three non-collinear points. Usually in this paper, two lines in a frame that contain the same points will be equal, so viewing a line as a set of points is not misleading. We will see in lemmas 3.2 and 3.5 below that this is true of affine planes.

§3. Non-finite axiomatisability. The rest of the paper is devoted to showing that the modal logic of affine planes is not finitely axiomatisable. We accomplish this in the following way. We build special quasi-affine structures which we call $\kappa$ configurations, where $\kappa$ is a cardinal. We will show, first, that for every finite $\kappa$, there is a finite $\kappa$-configuration that is not the bounded morphic image of an affine plane. Here, we make use of the Bruck-Ryser theorem in projective geometry. Second, we will show that any countable $\omega$-configuration is the bounded morphic image of an affine plane. With these results in hand, we then use first-order compactness to establish the non-finite axiomatisability result.

Definition 3.1. Let $\kappa$ be a cardinal. A frame $\mathscr{C}=(P, L, \varepsilon, \|)$ is said to be a $\kappa$-configuration if the following hold.

K1 For every $s, t \in P$, there are at least $\kappa$ lines $l \in L$ with $s, t \varepsilon l$.

$\mathrm{K} 2 \mathrm{~A} 2$ of definition 2.6 ('two lines are parallel iff they are equal or disjoint').

$\mathrm{K} 3 \mathrm{~A} 3$ of definition 2.6 ('there is a unique line through any given point parallel to any given line').

K4 $L \neq \emptyset$, and for any $l \in L$, there is $s \in P$ such that $\neg(s \& l)$.

A configuration is a $\kappa$-configuration for some $\kappa$ (i.e., a 0 -configuration).

Obviously, if $\kappa<\lambda$ then any $\lambda$-configuration is a $\kappa$-configuration. We list some other simple facts about configurations.

Lemma 3.2. Any affine plane is a 1-configuration.

Proof. By A1, K1 clearly holds when $s \neq t$. A4 ensures that there are at least two points, and $\mathrm{K} 1$ for the case $s=t$ follows from this and A1. We check K4. $L \neq \emptyset$ by A4 and A1. For any line $l$, at least one of the non-collinear points given by $\mathrm{A} 4$ cannot be on $l$.

In fact, $\mathrm{K} 4$ is equivalent to $\mathrm{A} 4$ in the presence of A1-A3. With only $\mathrm{K} 1-\mathrm{K} 3$, it is weaker. 
LEMMA 3.3. II is an equivalence relation on the set of lines in any configuration.

Proof. Reflexivity and symmetry are clear. For transitivity, suppose that $l, m, n$ are lines with $l\|m\| n$. If $l, n$ are disjoint, then $l \| n$ by $\mathrm{K} 2$. If they have a common point, say $s$, then by K3 they are equal, since they are parallel to $m$ and contain $s$. By K2 we again obtain $l \| n$.

The equivalence classes of $\|$ will be called parallel classes.

Lemma 3.4. Any line in a 1-configuration contains a point.

Proof. Using K4, take a line $l$ and a point $s$ not on $l$. By K3, there is a line $m \| l$ with $s \varepsilon m$. By K4, there is a point $t$ not on $m$. We are working in a 1-configuration, so by $\mathrm{K} 1$ there is a line $n$ with $s, t \varepsilon n$. So $n \neq m$. If some line had no points, then by $\mathrm{K} 2, n, m$ would both be parallel to it, which violates $\mathrm{K} 3$ since they both contain $s$.

Lemma 3.5. Any two lines in a 1-configuration that contain the same points are equal.

Proof. We actually show that in any configuration, any two 'non-empty' lines containing the same points are equal. The result then follows from lemma 3.4. So let $l, m$ be lines containing the same points, and suppose that there is a point $s$ on $l$, and hence on $m$. By $\mathrm{K} 4$, there is a point $t$ not on $l$. By $\mathrm{K} 3$, there is a line $n$ containing $t$ and parallel to $l$. Since $n \neq l, \mathrm{~K} 2$ implies that $n$ and $l$ are disjoint. So $n, m$ are also disjoint, and hence (by K2) parallel. We conclude that $l, m$ contain $s$ and are parallel to $n$. By $\mathrm{K} 3, l=m$.

Proposition 3.6. For every $k<\omega$, there is a finite $k$-configuration $\mathscr{C}_{k}$ with exactly c parallel classes, where $c=2 \cdot 3^{2 e+1}+1$ for some integer $e$.

Proof. Pick integers $c, d, e$ with $k \leq 2^{d-2}, 4 k d^{2} \leq 2 \cdot 3^{2 e+1}+1=c$, and $c \leq 2^{d-1}$. Then $c, d>0$. Take any set $P$ with $|P|=2 d$, and put $[P]^{d}=\{l \subseteq P:|l|=d\}$. Choose $L \subseteq[P]^{d}$ satisfying the following:

1. $l \in L \Rightarrow P \backslash l \in L$. (Note that $P \backslash l \in[P]^{d}$.)

2. For each $s, t \in P$, there are at least $k$ sets $l \in L$ with $s, t \in l$. This is possible since $\left|\left\{l \in[P]^{d}: s, t \in l\right\}\right| \geq\left(\begin{array}{c}2 d-2 \\ d-2\end{array}\right) \geq 2^{d-2} \geq k$, so we may simply choose $k$ sets in $[P]^{d}$ containing $s, t$ to add to $L$, for each $s, t \in P$. There is no problem if the same set is chosen for several pairs $s, t$. We need to pick a total of at most $(2 d)^{2} k$ sets, plus their complements (because of clause 1 ).

3. $|L|=2 c$. So far, $|L| \leq 8 k d^{2} \leq 2 c$. Simply add more $l \in[P]^{d}$ (and their complements) to $L$ until $|L|=2 c$. This is possible since $\left|[P]^{d}\right|=\left(\begin{array}{c}2 d \\ d\end{array}\right) \geq 2^{d} \geq$ $2 c$.

For $s \in P$ and $l \in L$, define $s \varepsilon l$ iff $s \in l$. For $l, m \in L$, define $l \| m$ iff $l=m$ or $l \cap m=\emptyset$. We check that $\mathscr{C}_{k}=(P, L, \varepsilon, \|)$ is as required. K1 of definition 3.1 holds by clause 2 . K2 holds by definition of $\|$. $L \neq \emptyset$ by clause 3 , and $P \backslash l \neq \emptyset$ for all $l \in L$, so K4 holds.

$\mathrm{K} 3$ and the final statement of the proposition will follow immediately if we show that the parallel class of an arbitrary line $l \in L$ is $\left\{l, l^{\prime}\right\}$, where $l^{\prime}=P \backslash l$. We have $l^{\prime} \in L$ by clause 1 . By definition of $\|$, we have $l \| l$ and $l^{\prime} \| l$. Now if $m \in L$ and $m \| l$, then either $m=l$ or $m \cap l=\emptyset$. In the latter case, $m \subseteq l^{\prime}$, and because $|m|=\left|l^{\prime}\right|=d$, we have $m=l^{\prime}$ as required. 
From now on, fix $k$-configurations $\mathscr{C}_{k}(k<\omega)$ as in the proposition. By replacing $\mathscr{C}_{0}$ by $\mathscr{C}_{1}$ if necessary, we may assume that each $\mathscr{C}_{k}$ is a 1 -configuration.

Proposition 3.7. For each $k, \mathscr{C}_{k}$ is not a homomorphic image (and so not a bounded morphic image) of an affine plane.

Proof. Suppose for contradiction that $\mathscr{A}$ is an affine plane and $f: \mathscr{A} \rightarrow \mathscr{C}_{k}$ is a surjective homomorphism. We write the relations of both $\mathscr{A}, \mathscr{C}_{k}$ as $\varepsilon, \|$.

Claim. For any lines $l, m$ of $\mathscr{A}$, we have $l\|m \Longleftrightarrow f(l)\| f(m)$.

ProOF OF ClAIM. The left to right direction $(\Rightarrow)$ is clear as $f$ is a homomorphism. For the opposite direction $(\Leftarrow)$, suppose for contradiction that $l, m$ are not parallel and yet $f(l) \| f(m)$. Since $\mathscr{A}$ is an affine plane, it has a point $s$ with $s \varepsilon l$, $s \varepsilon m$. As $f$ is a homomorphism, $f(s) \varepsilon f(l)$ and $f(s) \varepsilon f(m)$, so we have $f(l)=f(m)$ by $\mathrm{K} 2$ of definition 3.1. By K4 and K3, the parallel class of $f(l)$ contains a line other than $f(l)$, and by surjectivity, such a line is of the form $f(n)$ for some line $n$ of $\mathscr{A}$. Since $\|$ is an equivalence relation on the lines of $\mathscr{A}, n$ cannot be parallel to both of $l, m$, so it has a point, say $t$, in common with one of them. But then, $f$ being a homomorphism implies $f(t) \varepsilon f(n)$ and $f(t) \varepsilon f(l)=f(m)$. Since by K2 these two lines are disjoint, this is a contradiction, and proves the claim.

It follows from the claim that $\mathscr{C}_{k}$ and $\mathscr{A}$ have the same number of parallel classes, namely, $2 \cdot 3^{2 e+1}+1$ for some integer $e$. So $\mathscr{A}$ has order $2 \cdot 3^{2 e+1}$. As is well known (see, e.g., [8, theorem 3.10]), any affine plane can be 'completed' to form a projective plane of the same order. Now the Bruck-Ryser theorem [5] implies that if $n \equiv 1$ or 2 $(\bmod 4)$ and there is a projective plane of order $n$, then $n$ is the sum of the squares of two integers. It is obvious that $2 \cdot 3^{2 e+1} \equiv 2(\bmod 4)$. Since the prime factorisation of $2 \cdot 3^{2 e+1}$ involves a prime $p \equiv 3(\bmod 4)$ with odd exponent, it follows from well known results of Fermat (see, e.g., [11, chapter XI] or [7, theorem 366]) that $2 \cdot 3^{2 e+1}$ is not the sum of two squares. From this contradiction we conclude that $\mathscr{C}_{k}$ is not a homomorphic image of an affine plane.

Proposition 3.8. Any countable $\omega$-configuration is a bounded morphic image of an affine plane.

Proof. Let $\mathscr{C}=(P, L, \varepsilon, \|)$ be a countable $\omega$-configuration. We will show that it is a bounded morphic image of an affine plane via a step by step construction similar to the one presented in [13] for projective planes. A network is a quintuple $N=\left(P^{\prime}, L^{\prime}, \varepsilon^{\prime}, \|^{\prime}, f\right)$, where $\left(P^{\prime}, L^{\prime}, \varepsilon^{\prime}, \|^{\prime}\right)$ is a frame and $f$ is a function mapping $P^{\prime}$ to $P$ and $L^{\prime}$ to $L$. We will build a chain of finite networks $N_{0} \subseteq N_{1} \subseteq \cdots$, where $N_{i}=\left(P_{i}, L_{i}, \varepsilon_{i}, \|_{i}, f_{i}\right)$ for each $i<\omega$, and $N_{i} \subseteq N_{j}$ denotes that $N_{j}$ is an extension of $N_{i}$, i.e., that $P_{i}$ and $L_{i}$ are subsets of $P_{j}$ and $L_{j}$, respectively; $\varepsilon_{i}$ is the restriction of $\varepsilon_{j}$ to $P_{i} \times L_{i} ; \|_{i}$ is the restriction of $\|_{j}$ to $L_{i} \times L_{i}$; and $f_{i}$ is the restriction of $f_{j}$ to $P_{i} \cup L_{i}$.

A triangle is a frame with exactly three lines, each line being parallel only to itself, and exactly three points, each pair of which are joined by exactly one line. We define $N_{0}$ to consist of a triangle with points $s_{0}, s_{1}, s_{2}$, say, and a map $f_{0}$ that maps its points to an arbitrary single point $s$ in $\mathscr{C}$, and its lines to three pairwise non-parallel lines through $s$ (existence is assured because $\mathscr{C}$ is an $\omega$-configuration). 
Each $N_{k}(k<\omega)$ will satisfy the following coherence conditions:

C1 For all points $s$ and lines $l$ of $N_{k}$, if $s \varepsilon_{k} l$ then $f_{k}(s) \varepsilon f_{k}(l)$.

C2 $l\left\|_{k} m \Longleftrightarrow f_{k}(l)\right\| f_{k}(m)$, for all lines $l, m$ of $N_{k}$.

C3 Distinct lines of $N_{k}$ have at most one common point.

C4 Distinct parallel lines of $N_{k}$ are disjoint.

C5 $s_{0}, s_{1}, s_{2}$ are non-collinear points of $N_{k}$.

Clearly, $N_{0}$ is a coherent network. However, it, and later networks $N_{k}$, may suffer from a number of defects, which we will need to repair. The possible defects are:

D1 $f_{k}$ failing any of the 'back' conditions B1, B2, and B3 in definition 2.4 (bounded morphism) for $\langle 01\rangle,\langle 10\rangle,\langle 11\rangle$,

D2 two points with no line joining them,

D3 the parallel axiom defect, namely, for a line $l$ and point $s$ not on $l$, there is no line through $s$ that is parallel to $l$,

D4 non-parallel lines with no point in common.

The way we repair a defect is by extending a network into another. We will now demonstrate how any defect of a coherent network $N_{k}$ can be repaired. There are a number of cases to consider depending on the type of the defect.

D1-defects. These come in three forms: B1, B2, and B3.

B1 a point $s \in P_{k}$ and a line $l^{\prime} \in L$ such that $f_{k}(s) \varepsilon l^{\prime}$ while there is no $l \in L_{k}$ such that $s \varepsilon_{k} l$ and $f_{k}(l)=l^{\prime}$.

Take a new line $l\left(l \notin L_{k}\right)$ and extend $N_{k}$ to $N_{k+1}$ as follows:

1. $P_{k+1}=P_{k}$,

2. $L_{k+1}=L_{k} \cup\{l\}$,

3. $\varepsilon_{k+1}=\varepsilon_{k} \cup\{(s, l)\}$,

4. $f_{k+1}=f_{k} \cup\left\{\left(l, l^{\prime}\right)\right\}$,

5. $\left\|_{k+1}=\right\|_{k} \cup\left\{(l, m),(m, l): m \in L_{k+1}, l^{\prime} \| f_{k+1}(m)\right\}$.

Clearly, $N_{k+1}$ is an extension of $N_{k}$ lacking the assumed defect. We check that $N_{k+1}$ is coherent. For condition $\mathrm{Cl}$, since $N_{k}$ is assumed coherent, we only have to check whether $f_{k+1}(s) \in f_{k+1}(l)$. This is true by construction. C2 holds by the definition of $\|_{k+1}$ and the coherence of $N_{k}$. Suppose that C3 is violated. By the definition of $N_{k+1}$ and the fact that $N_{k}$ satisfies C3, this means that the new line $l$ must be one of the culprits. But $l$ intersects only one point $(s)$ and is therefore absolved of any blame. We conclude that C3 holds as well. Suppose $\mathrm{C} 4$ is violated. By the coherence of $N_{k}$, we must infer that $l$ is involved, and since $l$ only goes through $s, s$ also is indicted. So there is a line $m$ of $N_{k}$ with $s \varepsilon_{k} m$ and $l \|_{k+1} m$. Therefore, $f_{k}(s) \varepsilon l^{\prime} \cap f_{k+1}(m)$ and $l^{\prime} \| f_{k+1}(m)$. Since $\mathscr{C}$ is a configuration, $f_{k+1}(m)=l^{\prime}$, and so there is already a line in $N_{k}$, namely $m$, with $s \varepsilon_{k} m$ and $f_{k}(m)=l^{\prime}$. So there was no B1 defect in the first place! Therefore, we conclude that $\mathrm{C} 4$ also holds. $\mathrm{C} 5$ is true because it holds for $N_{k}$ and there is only one point on the new line $l$, so not all of $s_{0}, s_{1}, s_{2}$ can be on it.

B2 a line $l \in L_{k}$ and a point $s^{\prime} \in P$ such that $s^{\prime} \varepsilon f_{k}(l)$, while there is no $s \in P_{k}$ such that $s \varepsilon_{k} l$ and $f_{k}(s)=s^{\prime}$. 
Take a new point $s\left(s \notin P_{k}\right)$ and extend $N_{k}$ to $N_{k+1}$ as follows:

1. $P_{k+1}=P_{k} \cup\{s\}$,

2. $L_{k+1}=L_{k}$,

3. $\varepsilon_{k+1}=\varepsilon_{k} \cup\{(s, l)\}$,

4. $\left\|_{k+1}=\right\|_{k}$,

5. $f_{k+1}=f_{k} \cup\left\{\left(s, s^{\prime}\right)\right\}$.

Clearly, $N_{k+1}$ is an extension of $N_{k}$ lacking the assumed defect. We check that the coherence conditions remain intact. For $\mathrm{C} 1$, the only new case is $f_{k+1}(s) \varepsilon f_{k+1}(l)$; but this is true by construction. $\mathrm{C} 2$ is immediate. Since $s$ is only incident with one line (l), C3 and C4 are preserved. C5 is true because it was true in $N_{k}$ and no points of $N_{k}$ have been added to lines.

B3 a line $n \in L_{k}$ and a line $l^{\prime} \in L$ such that $f_{k}(n) \| l^{\prime}$ while there is no line $l \in L_{k}$ with $n \|_{k} l$ and $f_{k}(l)=l^{\prime}$.

Take a new line $l\left(l \notin L_{k}\right)$ and extend $N_{k}$ to $N_{k+1}$ as follows:

1. $P_{k+1}=P_{k}$,

2. $L_{k+1}=L_{k} \cup\{l\}$,

3. $\varepsilon_{k+1}=\varepsilon_{k}$,

4. $\left\|_{k+1}=\right\|_{k} \cup\left\{(m, l),(l, m): m \in L_{k+1}, m=l\right.$ or $\left.m \|_{k} n\right\}$,

5. $f_{k+1}=f_{k} \cup\left\{\left(l, l^{\prime}\right)\right\}$.

Clearly, $N_{k+1}$ is an extension of $N_{k}$ lacking the assumed defect. C1, C3, C4, and C5 are unaffected since $\varepsilon_{k+1}=\varepsilon_{k}$, and $\mathrm{C} 2$ follows from the definition of $\|_{k+1}$ and the coherence of $N_{k}$.

D2-defects. This case is the crux of the proof. Assume there are two distinct points $s$ and $t$ of $N_{k}$ with no line joining them. Add a new line $l\left(l \notin L_{k}\right)$ joining $s, t$, and let $f_{k+1}$ map $l$ to a line $l^{\prime}$ of $\mathscr{C}$ containing $f_{k}(s), f_{k}(t)$ and whose parallel class has not been used so far: i.e., there is no line $m$ in $N_{k}$ with $f_{k}(m) \| l^{\prime}$. This is possible as $N_{k}$ is finite, while because $\mathscr{C}$ is an $\omega$-configuration there are $\omega$ pairwise non-parallel $l^{\prime} \in L$ with $f_{k}(s), f_{k}(t) \varepsilon l^{\prime}$ (see $\mathrm{K} 1$ and $\mathrm{K} 2$ of definition 3.1). It avoids there already being a line through $s$ but not $t$ which maps by $f_{k}$ to $l^{\prime}$ and so (by $\mathrm{C} 2$ ) has to be parallel to $l$.

More precisely, we extend $N_{k}$ to $N_{k+1}$ as follows:

1. $P_{k+1}=P_{k}$,

2. $L_{k+1}=L_{k} \cup\{l\}$,

3. $\varepsilon_{k+1}=\varepsilon_{k} \cup\{(s, l),(t, l)\}$,

4. $\left\|_{k+1}=\right\|_{k} \cup\{(l, l)\}$,

5. $f_{k+1}=f_{k} \cup\left\{\left(l, l^{\prime}\right)\right\}$.

$N_{k+1}$ is an extension of $N_{k}$ lacking the assumed defect. We will now check that none of the coherence conditions have been broken. For $\mathrm{C} 1$, we have to check that $f_{k+1}(s) \varepsilon f_{k+1}(l)$ and $f_{k+1}(t) \varepsilon f_{k+1}(l)$; but this follows from our choice of $l^{\prime}$. $\mathrm{C} 2$ also follows immediately from our choice of $l^{\prime}$. Now suppose $\mathrm{C} 3$ is violated; by the coherence of $N_{k}$ the new line $l$ must be involved. As $l$ is only incident with the points $s$ and $t$, there must be another line $m$ that goes through these points - but then $s$ and $t$ could not constitute a D2-defect! C4 holds because $N_{k}$ is coherent and $l$ is only parallel to itself. C5 is preserved because $l$ contains only two points. 
D3-defects. Given a line $n$ and a point $s$ not incident with $n$, assume there is no line through $s$ which is parallel to $n$. By K3 of definition 3.1, we know that there is a line $l^{\prime}$ of $\mathscr{C}$ that goes through $f_{k}(s)$ and is parallel to $f_{k}(n)$. So take a new line $l$ $\left(l \notin L_{k}\right)$ and define the extension $N_{k+1}$ of $N_{k}$ as follows:

1. $P_{k+1}=P_{k}$,

2. $L_{k+1}=L_{k} \cup\{l\}$,

3. $\varepsilon_{k+1}=\varepsilon_{k} \cup\{(s, l)\}$,

4. $\left\|_{k+1}=\right\|_{k} \cup\left\{(m, l),(l, m): m \in L_{k+1}, m=l\right.$ or $\left.m \|_{k} n\right\}$,

5. $f_{k+1}=f_{k} \cup\left\{\left(l, l^{\prime}\right)\right\}$.

$N_{k+1}$ is an extension of $N_{k}$ lacking the assumed defect. For $\mathrm{C} 1$ we have to check that $f_{k+1}(s) \in f_{k+1}(l)$, but this is so by our assumption on $s$ and $l^{\prime}$. It is not difficult to see that $\mathrm{C} 2$ also holds by our definition of $\|_{k+1}$ and the coherence of $N_{k}$. So suppose $\mathrm{C} 3$ is violated. By the coherence of $N_{k}$, the new line $l$ must be the cause; but $l$ is only incident with the point $s$, and therefore cannot cause any problems with respect to C3. Suppose C4 is violated. Again, by the coherence of $N_{k}$, the new line $l$ must be involved. We must therefore conclude that there is a distinct line $m$ parallel to $l$ that also intersects $l$. By definition of $\|_{k+1}$, this means that $m \|_{k} n$. Since $l$ is only incident with the point $s, m$ must also be incident with $s$. So $n$ and $s$ did not constitute a D3-defect after all! This proves C4. C5 is clearly satisfied as $l$ contains only one point.

D4-defects. Assume $n$ and $l$ are two non-parallel lines in $N_{k}$ that do not intersect. As $N_{k}$ is coherent, $\mathrm{C} 2$ shows that $f_{k}(n)$ and $f_{k}(l)$ are not parallel in $\mathscr{C}$. By K2 of definition 3.1, we know that there is a point $s^{\prime} \varepsilon f_{k}(n) \cap f_{k}(l)$. So take a new point $s\left(s \notin P_{k}\right)$, and define the extension $N_{k+1}$ of $N_{k}$ as follows:

1. $P_{k+1}=P_{k} \cup\{s\}$,

2. $L_{k+1}=L_{k}$,

3. $\varepsilon_{k+1}=\varepsilon_{k} \cup\{(s, n),(s, l)\}$,

4. $\left\|_{k+1}=\right\|_{k}$,

5. $f_{k+1}=f_{k} \cup\left\{\left(s, s^{\prime}\right)\right\}$.

$N_{k+1}$ is an extension of $N_{k}$ lacking the assumed defect. We check coherence. For $\mathrm{C} 1$, we need to show that $f_{k+1}(s) \varepsilon f_{k+1}(n)$ and $f_{k+1}(s) \varepsilon f_{k+1}(l)$; but this is obviously the case by construction. C2 is satisfied by the coherence of $N_{k}$ since $\left\|_{k+1}=\right\|_{k}$. Suppose C3 is violated. By the coherence of $N_{k}$ the new point $s$ must be involved. But $s$ lies only on the lines $n$ and $l$; if these two lines intersect at a different point then they could not have constituted a D4-defect in the first place. Finally, suppose $\mathrm{C} 4$ is violated. By the coherence of $N_{k}$, we know that $s$ must be involved. But $s$ only intersects $n$ and $l$, which are non-parallel, and therefore cannot cause any violation of C4. C5 is satisfied as no points of $N_{k}$ were added to lines.

We have shown that any defect in any $N_{k}$ can be repaired in an extension of $N_{k}$. Now using standard combinatorics we construct a sequence $\left(N_{k}\right)_{k<\omega}$ of coherent networks such that $N_{j}$ extends $N_{i}$ whenever $j>i$, and every defect of $N_{i}$ is repaired in $N_{j}$ for some $j>i$. The 'standard combinatorics' needed to make sure that every defect of every network $N_{i}$ will be repaired at some later stage is completely analogous to a proof in [12, section 2] and makes use of the fact that $\mathscr{C}$ is countable. Now put $P_{\omega}=\bigcup_{k<\omega} P_{k}, L_{\omega}=\bigcup_{k<\omega} L_{k}, \varepsilon_{\omega}=\bigcup_{k<\omega} \varepsilon_{k}$, and $\left\|_{\omega}=\bigcup_{k<\omega}\right\|_{k}$. Let 
$\mathscr{A}=\left(P_{\omega}, L_{\omega}, \varepsilon_{\omega}, \|_{\omega}\right)$. Also put $f=\bigcup_{k<\omega} f_{k}$. As the coherence conditions are clearly preserved under unions of chains, $(\mathscr{A}, f)$ is a coherent network with no defects. In order to finish the proof of the proposition, we establish the following claims.

Claim 1. The mapping $f: \mathscr{A} \rightarrow \mathscr{C}$ is a surjective bounded morphism.

Proof of Claim 1. That $f$ is a homomorphism follows from $\mathrm{C} 1$ and $\mathrm{C} 2$. The back conditions hold because $(\mathscr{A}, f)$ has no D1-defects. It remains to show that $f$ is surjective. Let $l$ be any line in $\mathscr{A}$. Then $f(l) \in r n g(f)$. By the 'back' condition B3, any line $l^{\prime}$ of $\mathscr{C}$ with $l^{\prime} \| f(l)$ is in $r n g(f)$. By the 'back' condition B2 and definition 3.1(K3), $P \subseteq r n g(f)$. Recalling from lemma 3.4 that any line in an $\omega$-configuration contains at least one point, by the 'back' condition B1 we also have $L \subseteq r n g(f)$. This establishes the claim.

Claim 2. $\mathscr{A}$ is an affine plane.

Proof of Claim 2. We check that $\mathscr{A}$ meets the conditions of definition 2.6. We will need that $\|_{\omega}$ is an equivalence relation on $L_{\omega}$. This follows from $\mathrm{C} 2$, since by lemma $3.3, \|$ is an equivalence relation on the lines of $\mathscr{C}$. Now, A1 ('any two distinct points lie on a unique line') holds by $\mathrm{C} 3$ and the lack of D2-defects. A2 ( $\Rightarrow$ direction) is true by C4. For the $\Leftarrow$ direction, let $l, m$ be lines of $\mathscr{A}$. If $l=m$, then $l \|_{\omega} m$ as $\|_{\omega}$ is reflexive. If $l, m$ have no point in common, then $l \|_{\omega} m$ since $\mathscr{A}$ has no D4 defects. So $\mathscr{A}$ satisfies A2. For A3, because $\mathscr{A}$ has no D3 defects, for any line $n$ and point $s$, there is a line through $s$ parallel to $n$. Suppose for contradiction that there are two distinct lines $l$ and $m$ passing through $s$ and parallel to $n$. As $\|_{\omega}$ is an equivalence relation, $l \|_{\omega} m$. But then we have two distinct parallel lines $l$ and $m$ that intersect at a point, namely $s$, thus violating $\mathrm{C} 4$ and contradicting the coherence of $\mathscr{A}$. By C5, $\mathscr{A}$ has three non-collinear points, so $\mathrm{A} 4$ is satisfied. This proves claim 2.

Thus we conclude that $\mathscr{A}$ is an affine plane and $\mathscr{C}$ is a bounded morphic image of $\mathscr{A}$, thereby establishing the truth of proposition 3.8.

We are nearly ready to establish our main result. We need three preliminary remarks.

REMARK 3.9. We recall the standard translation of modal formulas to first-order ones. See, e.g., [4, definition 2.45], or [6, p.122]. We tailor it to our two-sorted system. For each $v \in V A R_{p} \cup V A R_{l}$, introduce a unary relation symbol $Q_{v}$. Let $\mathscr{L}$ be the signature consisting of these symbols together with binary relation symbols $\varepsilon$ and $\|$. Then for each modal formula $\varphi$ and each first-order variable $x$, we define a first-order $\mathscr{L}$-formula $\varphi^{x}$ by induction on $\varphi$, as follows. $v^{x}=Q_{v}(x) ;(\neg \varphi)^{x}=$ $\neg \varphi^{x} ;(\varphi \wedge \psi)^{x}=\varphi^{x} \wedge \psi^{x} ;([01] \lambda)^{x}=\forall y\left(x \varepsilon y \rightarrow \lambda^{y}\right) ;([10] \pi)^{x}=\forall y\left(y \varepsilon x \rightarrow \pi^{y}\right)$; and $([11] \lambda)^{x}=\forall y\left(x \| y \rightarrow \lambda^{y}\right)$. Here, $\pi \in \Pi, \lambda \in \Lambda$, and $y$ is any variable other than $x$. Any modal model $\mathscr{M}=(P, L, \varepsilon, \|, V)$ can be viewed as a first-order $\mathscr{L}$ structure $M$ with domain $P \cup L$, with $\left(Q_{v}\right)^{M}=V(v)$ for each $v$, and with $\varepsilon$, $\|$ interpreted as in $\mathscr{M}$. If the frame of $\mathscr{M}$ is a configuration, $P$ and $L$ are definable by $\neg(x \| x)$ and $x \| x$, respectively. Then, for every point formula $\pi$, the statement that $\mathscr{M}, s \models \pi$ for some $s \in P$ is equivalent to $M \models \widehat{\pi}$, where $\widehat{\pi}=\exists x\left(\neg(x \| x) \wedge \pi^{x}\right)$, and similarly for line formulas. 
ReMARK 3.10. We will be using Jankov-Fine formulas. See [6, chapter 9] or [4, theorem 3.21] for information. First, define the following abbreviations:

$$
\begin{aligned}
& {[\cdot] \pi=[01][10] \pi \text {, for any point formula } \pi \text {, }} \\
& {[-] \lambda=[01][11] \lambda \text {, for any line formula } \lambda \text {. }}
\end{aligned}
$$

These will serve as 'universal modalities': in any model whose frame is a 1-configuration, $[\cdot] \pi$ is true at a point $s$ iff $\pi$ is true at all points, and $[-] \lambda$ is true at $s$ iff $\lambda$ is true at all lines. Let $\langle\cdot\rangle$ and $\langle-\rangle$ denote the corresponding diamonds: i.e., $\langle\cdot\rangle \pi=\neg[\cdot] \neg \pi$, etc.

Now let $k<\omega$, and write the $\mathscr{C}_{k}$ that we obtained from proposition 3.6 as $\left(P_{k}, L_{k}, \varepsilon_{k}, \|_{k}\right)$. As $\mathscr{C}_{k}$ is finite and we have definable universal modalities for it, we can write a 'Jankov-Fine formula' $\xi_{k}$ describing its entire structure. Enumerate the points and lines of $\mathscr{C}_{k}$ as $P_{k}=\left\{s_{0}, \ldots, s_{m}\right\}$ and $L_{k}=\left\{l_{0}, \ldots, l_{w}\right\}$. Associate with each point $s_{i}$ and line $l_{j}$ a distinct point propositional letter $p_{i} \in V A R_{p}$ and a distinct line propositional letter $a_{j} \in V A R_{l}$, respectively. Now define $\xi_{k}$ (a point formula) to be the conjunction of the following formulas:

1. $[\cdot]\left(p_{0} \vee \cdots \vee p_{m}\right) \wedge \bigwedge_{i \leq m}\langle\cdot\rangle p_{i}$.

2. $[-]\left(a_{0} \vee \cdots \vee a_{w}\right) \wedge \bigwedge_{j \leq w}\langle-\rangle a_{j}$.

3. [·] $\left(p_{i} \rightarrow \neg p_{j}\right)$ for each $i, j$ with $i \neq j$.

4. [-] $\left(a_{i} \rightarrow \neg a_{j}\right)$ for each $i, j$ with $i \neq j$.

5. [.] $\left(p_{i} \rightarrow\langle 01\rangle a_{j}\right)$ for each $i, j$ such that $s_{i} \varepsilon_{k} l_{j}$.

6. [.] $\left(p_{i} \rightarrow \neg\langle 01\rangle a_{j}\right)$ for each $i, j$ such that $\neg\left(s_{i} \varepsilon_{k} l_{j}\right)$.

7. $[-]\left(a_{i} \rightarrow\langle 10\rangle p_{j}\right)$ for each $i, j$ such that $s_{j} \varepsilon_{k} l_{i}$.

8. [-] $\left(a_{i} \rightarrow \neg\langle 10\rangle p_{j}\right)$ for each $i, j$ such that $\neg\left(s_{j} \varepsilon_{k} l_{i}\right)$.

9. $[-]\left(a_{i} \rightarrow\langle 11\rangle a_{j}\right)$ for each $i, j$ such that $l_{i} \|_{k} l_{j}$.

10. $[-]\left(a_{i} \rightarrow \neg\langle 11\rangle a_{j}\right)$ for each $i, j$, such that $\neg\left(l_{i} \|_{k} l_{j}\right)$.

Let $V$ be any valuation on $\mathscr{C}_{k}$ such that $V\left(p_{i}\right)=\left\{s_{i}\right\}(i \leq m)$ and $V\left(a_{i}\right)=\left\{l_{i}\right\}$ $(i \leq w)$. Plainly, $\left(\mathscr{C}_{k}, V\right), s \models \xi_{k}$ for any $s \in P_{k}$, so $\xi_{k}$ is satisfiable in $\mathscr{C}_{k}$.

On the other hand, suppose that $\xi_{k}$ is satisfiable in an affine plane $\mathscr{A}$. Let $\left(\mathscr{A}, V^{\prime}\right)$ be a model in which $\xi_{k}$ is true at some point. Define a map $f$ by stipulating that for each point $s$ of $\mathscr{A}, f(s)$ is the unique $s_{i} \in P_{k}$ with $s \in V^{\prime}\left(p_{i}\right)$, and similarly for lines. Then a standard argument shows that $f: \mathscr{A} \rightarrow \mathscr{C}_{k}$ is a well defined surjective bounded morphism. Since by proposition $3.7, \mathscr{C}_{k}$ is not the bounded morphic image of any affine plane, we conclude that $\neg \xi_{k}$ is valid in every affine plane.

REMARK 3.11. We will assume the following rules of inference: modus ponens (from $\varphi$ and $\varphi \rightarrow \psi$ derive $\psi$ ), generalisation (from $\pi \in \Pi$ derive [10] $\pi$, and from $\lambda \in \Lambda$ derive [01] $\lambda$ and [11] $\lambda$ ), and substitution (from $\varphi$, derive any formula obtained by replacing any occurrence in $\varphi$ of a variable in $V A R_{p}$ by an arbitrary point formula, and similarly for $V A R_{l}$ ). A set $\Phi$ of modal formulas is said to axiomatise the logic of affine planes if the set of formulas valid in all affine planes is precisely the smallest set of formulas containing $\Phi$ and closed under these inference rules (i.e., the set of formulas derivable from $\Phi$ ).

We can now prove our main result. The proof follows a well-trodden path. 
THEOREM 3.12. The modal logic of affine planes is not finitely axiomatisable.

Proof. Assume for contradiction that there is a finite set $\Phi$ of formulas that axiomatises the modal logic of affine planes. Let $k<\omega$. We know from remark 3.10 that $\neg \xi_{k}$ is valid in affine planes, so $\neg \xi_{k}$ is derivable from $\Phi$. If every $\varphi \in \Phi$ were valid in $\mathscr{C}_{k}$, then since the inference rules clearly preserve frame validity, $\neg \xi_{k}$ would also be valid in $\mathscr{C}_{k}$. Since we know from remark 3.10 that it is not, we deduce that there is a model $\mathscr{M}_{k}$ with frame $\mathscr{C}_{k}$ in which some $\varphi \in \Phi$ is false at some point or line. Let $\mathscr{L}$ be the signature and $M_{k}$ the first-order $\mathscr{L}$-structure obtained from $\mathscr{M}_{k}$ as in remark 3.9, and let $\theta=\bigvee_{\varphi \in \Phi} \widehat{\neg \varphi}$. Then $M_{k} \models \theta$. This holds for all $k$. Now for each $k<\omega$, we can write a first-order $\mathscr{L}$-sentence $\chi_{k}$ that is true in an $\mathscr{L}$-structure $M$ iff $M$ is the first-order counterpart of a model $\mathscr{M}$ whose frame is a $k$-configuration. Thus, $M_{k} \models \chi_{l}$ for all $l \leq k$. It follows by compactness that the $\mathscr{L}$-theory $\{\theta\} \cup\left\{\chi_{k}: k<\omega\right\}$ is consistent. Let $M$ be a countable model of it. As $M \models \chi_{k}$ for all finite $k$, there is a modal model $\mathscr{M}=(\mathscr{F}, V)$ of which $M$ is the first-order counterpart, and $\mathscr{F}$ is an $\omega$-configuration. By proposition $3.8, \mathscr{F}$ is the bounded morphic image of some affine plane $\mathscr{A}$. Now $\Phi$ axiomatises the logic of affine planes, so all its formulas are valid in $\mathscr{A}$. Since bounded morphisms preserve validity (remark 2.5), they are also valid in $\mathscr{F}$. But $M \models \theta$, so at least one of them is not valid in $\mathscr{F}$. This is a contradiction, and we therefore conclude that the modal logic of affine planes is not finitely axiomatisable.

The result holds for any inference rules that preserve validity in a configuration.

As far as we know, the fundamental problems of finding a transparent explicit axiomatisation of affine planes, and determining the decidability and (if decidable) the complexity of the logic of affine planes, remain open. Whether the logic of affine planes has the finite model property is also not known to us.

\section{REFERENCES}

[1] M. Aiello, I. Pratt-Hartmann, and J. van Benthem (editors), Handbook of spatial logics, SpringerVerlag, 2007.

[2] P. Balbiani, L. Fariñas del Cerro, T. Tinchev, and D. Vakarelov, Modal logics for incidence geometries, Journal of Logic and Computation, vol. 7 (1997), no. 1, pp. 59-78.

[3] P. BALBIANi and V. Goranko, Modal logics for parallelism, orthogonality, and affine geometries, Journal of Applied Non-Classical Logics, vol. 12 (2002), no. 3-4, pp. 365-398.

[4] P. Blackburn, M. De Rijke, and Y. Venema, Modal logic, Tracts in Theoretical Computer Science, Cambridge University Press, 2001.

[5] R. H. BRUCK and H. J. RYSER, The nonexistence of certain finite projective planes, Canadian Journal of Mathematics, vol. 1 (1949), pp. 88-93.

[6] A. Chagrov and M. Zakharyaschev, Modal logic, Clarendon Press, 1997.

[7] G. H. Hardy and E. M. Wright, An introduction to the theory of numbers, Clarendon Press, 1979, 5th edition.

[8] D. R. Hughes and F. C. PIPER, Projective planes, Graduate Texts in Mathematics, Springer-Verlag, 1973. 2007.

[9] A. Hussain, Some applications for extended modal logics, Ph.D. thesis, University of London,

[10] J. D. Monk, On representable relation algebras, Michigan Mathematics Journal, vol. 11 (1964), pp. 207-210.

[11] W. SIERPIŃSKI, Elementary theory of numbers, Państwowe Wydawnictwo Naukowe, Warszawa, 1964.

[12] Y Venema, Cylindric modal logic, this Journal, vol. 60 (1995), pp. 591-623. 
[13] Y. Venema, Points, lines and diamonds: a two-sorted modal logic for projective planes, Journal of Logic and Computation, vol. 9 (1999), pp. 601-621.

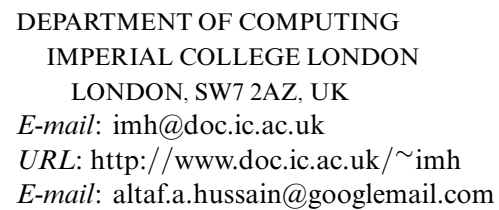

